Ann. Génét. Sél. anim., I977, 9 (2), I47-I50.

\title{
Anomalies chromosomiques chez les embryons de poulet issus de sperme âgé
}

\author{
C.-P. POPESCU et P. MÉRAT \\ avec la collaboration technique de Mime Jeannine Boscher \\ Laboratoire de Génétique factorielle, \\ Centre national de Recherches zootechniques, I.N.R.A., \\ 78350 Jouy-en-Josas
}

\begin{abstract}
Résumé
La présence de divers types d'anomalies chromosomiques a été examinée sur un total de I02 embryons issus de huit pères d'une lignée Leghorn naine. Ces embryons provenaient d'œufs collectés à partir du retrait des mâles d'avec les poules, jusqu'à disparition des œufs fertiles.

Les résultats suggèrent la possibilité d'une augmentation du pourcentage global d'anomalies chromosomiques parmi les embryons issus de sperme " âgé ".
\end{abstract}

\section{Introduction}

Chez les volailles en reproduction naturelle, après enlèvement d'un coq d'avec les poules auxquelles il est apparié, ou après une insémination artificielle, la femelle peut pondre des œufs fécondés pendant encore deux à trois semaines, par suite d'une survie prolongée des spermatozoïdes dans l'oviducte. Plusieurs auteurs ont signalé la baisse graduelle de fertilité (pourcentage d'ovules fécondée) qui s'observe dans ces conditions. Certains mentionnent, de plus, une augmentation de la mortalité embryonnaire. C'est le cas de Dunn (I927), Nalbandov et CARD (I943), Kosin (I943), Hale (I955), Parker (I950), Carter et al. (I957) et d'autres passés en revue par LANDAUER (I967), et plus récemment de LoDGE et al. (I97I). Cette mortalité embryonnaire accrue s'accompagnerait souvent d'après DHARMARAJAN (I950) d'anomalies anatomiques visibles.

LODGE et al. (I974) suggèrent d'autre part une augmentation de la proportion d'anomalies chromosomiques de divers ordres parmi des embryons de poulets issus de sperme " âgé ". 
La présente note concerne également une étude cytogénétique préliminaire sur des embryons issus de spermatozoïdes ayant séjourné plus ou moins longtemps dans l'oviducte de la poule.

\section{Matériel et méthodes}

En I974, huit coqs d'une souche Leghorn nanifiée (gène $d w$ ) ont été mis en reproduction chacun avec 5 femelles de la même souche. Après enlèvement de ces coqs (le 02-09-74) tous les œufs ont été collectés et mis en incubation jour par jour, jusqu'au $23^{\mathrm{e}}$ jour inclus suivant cet enlèvement (les œufs étant tous infertiles depuis le $2 \mathrm{I}^{\mathrm{e}} \mathrm{j}$ inclus). L'origine pedigree et la date de ponte étaient notées sur chaque œuf.

Les préparations chromosomiques ont été faites après 40 heures d'incubation, selon la méthode de FECHheimer et al. (1970). Les lames obtenues, généralement deux par embryon, ont été colorées au Giemsa et montées. Deux à cinq métaphases ont été photographiées pour chaque embryon. L'étude des chromosomes a été faite sur les images photographiques et pour certaines cellules on a établi le caryotype.

\section{Résultats}

Au total, 262 œufs ont été obtenus, dont II3 " clairs " (non fécondés) et I49 montrant un embryon en début de développement ou au moins quelques cellules. Parmi ces derniers, I02 embryons ont présenté plusieurs cellules en division permettant l'établissement de leur caryotype.

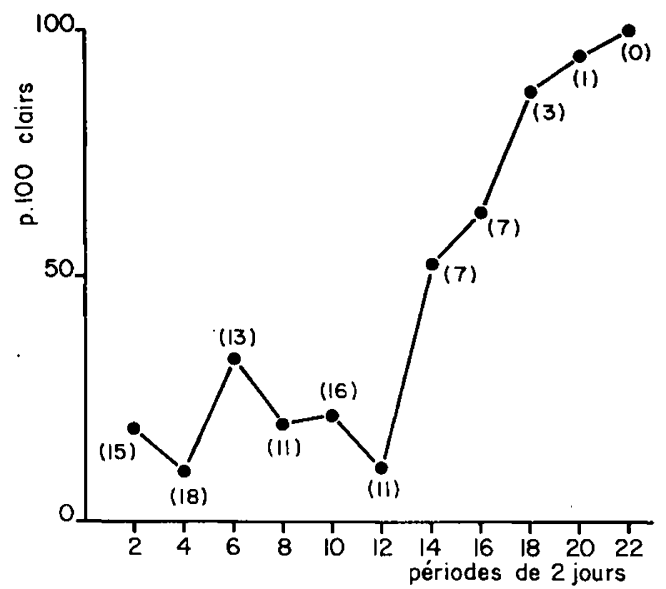

FJg. I. - Pourcentage d'eufs fertiles après le retrait des coqs (*)

Per cent infertile eggs after removal of coks

(*) Y,es nombres d'embryons obtenus sont indiqués entre parenthèses. Numbers of embryos obtained are mentioned within brackets. 
La figure I montre l'évolution du pourcentage d'œufs infertiles après le retrait des coqs (présentée pour la commodité par période de $2 \mathrm{j}$ ) : ce pourcentage augmente rapidement $d u{ }_{1}{ }^{e}$ au $2 \mathrm{I}^{\mathrm{e}}$ jour, à partir duquel il atteint $\mathrm{I} O 0 \mathrm{p}$. Ioo. Les nombres d'embryons obtenus par période sont indiqués entre parenthèses sur la figure.

Par contre, parmi les embryons obtenus, la proportion observable au point de vue cytogénétique, ne paraît pas différer nettement du début à la fin de la période considérée.

Sur les Io2 embryons examinés pour leur caryotype, II au total (soit Io,8 p. Ioo) ont été trouvés porteurs d'une anomalie décelable. Le tableau I montre leur nombre par type d'anomalie rencontrée. Les embryons sont issus de $S$ mères.

TABLEAU I

Types d'anomalies chromosomiques rencontrées parmi les embryons

Types of chromosomal abnormalities observed in the embryos

\begin{tabular}{|c|c|}
\hline $\begin{array}{c}\text { Type d'anomalie } \\
\text { Type of abnormality }\end{array}$ & $\begin{array}{l}\text { Nombre d'embryons } \\
\text { Number of embryos }\end{array}$ \\
\hline 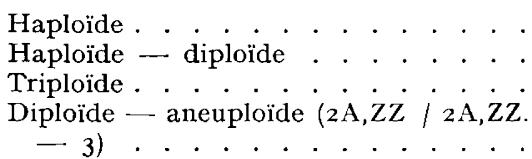 & $\begin{array}{l}4 \\
5 \\
I\end{array}$ \\
\hline
\end{tabular}

La répartition chronologique des embryons anormaux suivant le temps écoulé depuis le retrait des coqs est la suivante : $\mathrm{I}$ issu d'un œuf récolté le $4^{\mathrm{e}}$ jour, après ce retrait; 3 le $7^{\mathrm{e}} ; 2$, le $\mathrm{II}^{\mathrm{e}}$, I respectivement le $\mathrm{I} 3^{\mathrm{e}}$, I $5^{\mathrm{e}}$ et $\mathrm{I} 6^{\mathrm{e}}$ jour, 2 le $\mathrm{I} 7^{\mathrm{e}}$ jour.

Ces anormaux semblent donc se situer davantage à la fin qu'au début de la période envisagée. Si l'on partage cette période en 2 (jusqu'au $9^{\mathrm{e}} \mathrm{j}$, après le $9^{\mathrm{e}} \mathrm{j}$ ) on rencontre dans la première période 4 anormaux sur 57 embryons étudiés (7 p. IOo) et dans la deuxième 7 anormaux sur 45 embryons ( 15,6 p. IOO). On peut aussi remarquer que jusqu'au $6^{\mathrm{e}}$ jour il n'y a qu'un anormal sur 39 embryons. Cependant, la différence entre les deux périodes n'est pas significative ( $\alpha^{2}$ corrigé égal à $\mathrm{I}, \mathrm{I} 3)$.

Si l'on se restreint aux embryons produits par des poules qui ont eu des œufs fécondés au-delà du $\mathrm{I} 2^{\mathrm{e}}$ jour, la tendance à une augmentation du taux d'anomalies au cours du vieillissement des spermatozoïdes semble plus marquée : I anormal sur I 9 embryons avant le $6^{\mathbf{e}}$ jour, I sur $\mathrm{I} 8$ entre le $6^{\mathbf{e}}$ et le Io $^{\mathbf{e}}$ jour, 7 sur $3^{8}$ au-delà du IO $^{\mathrm{e}}$ jour.

\section{Discussion}

La proportion d'embryons porteurs d'anomalies chromosomiques dans les données présentes paraît à première vue plus élevée que les estimations données par BLOom (I974) sur des échantillons plus nombreux. Elle est par contre du même ordre que celle donnée par Miller et al. (I976). Cependant la partie de nos données comprenant des embryons obtenus avec du sperme "âgé " ne correspond pas à 
des conditions comparables à celles des auteurs précédents et est à exclure de la comparaison. Étant donnée d'autre part la taille limitée de l'échantillon restant, on ne peut conclure si la fréquence des anomalies chromosomiques dans notre population diffère de celles obtenues antérieurement sur des souches Leghorn.

Les anomalies prédominantes en nombre dans nos données, haploïdes et mosaïques haploïdes-diploïdes, sont les plus fréquemment trouvées dans la littérature (BLoOM, I972, I974; Miller et al., I976).

La réalité d'une augmentation de fréquence des aberrations chromosomiques chez les embryons issus de spermatozoïdes conservés longtemps dans les voies génitales de la poule ne peut être prouvée définitivement par nos résultats. Cependant, ceux-ci vont dans le même sens que ceux de LoDGE et FechHeImer (I974) et suggèrent donc cette possibilité.

Reģu pour publication en mai 1977.

\section{Summary \\ Chromosomic abnormalities in chicken embryos from aged semen}

The presence of various types of chromosomal abnormalities was investigated on a total of I02 embryos issued from 8 sires of a Dwarf Leghorn strain. These embryos came from eggs collected after removal of males, till disappearance of any fertile egg.

Results suggest possible increase of total per cent chromosome abnormalities among embryos produced by " aged " sperm.

\section{Références bibliographiques}

Bцоом S. E., 1972. Chromosome abnormalities in chicken (Gallus domesticus) embryos: Types, frequencies and phonotypic effects. Chromosoma, 37, 309-326.

Bloom S. E., I974. The origin and phenotypic effects of chromosome abnormalities in avian embryos. I $5^{\mathrm{e}}$ congrès mondial d'aviculture, 316-321.

Carter R. D., McCartney M. G., Chamberlin V. D., Wyne J. W., i957. The effect of storage time and temperature on fertilizing capacity of turkey semen. Poult. Sci., 36, 6r8-62r.

Dharmarajan M., r950. Effect on the embryo of staleness of the sperm at the time of fertilization in the domestic hen. Nature, 165, 398.

Dunn L. C., I927. Selective fertilization in fowls. Poult. Sci., 6, 20I-2I4.

Fechietmer N. S., Lodge J. R., Miller R. C., 1970. Sex proportion of domestic chickens at $\mathrm{I} 6$ hours of incubation. J. reprod. fertil., 23, 365-367.

Hale E. B., I955. Duration of fertility and hatchability following natural matings in turkeys. Poult. Sci., 34, 228-233.

Kosin I. L., 1947. Preliminary results of a study on the effect of in vivo aging of spermatozoa on the viability of chicken embryos. Poult. Sci., 26, 548 (abstr.).

LANDAUER W., I967. The hatchability of chicken eggs as influenced by environment and heredity. Stors Agric. Exp. Sta. Monogr., 1, I32-137.

Lodge J. R., Ax R. L., Fechheimer N. S., 1974. Chromosome aberrations in embryos from in vivo aged chicken sperm. Poult. Sci., 53, 1816-1819.

Lodge J. R., FechHeimer N. S., JAAP R. G., 197r. The relationship of in vivo sperm storage interval to fertility and embryonic survival in the chicken. Biol. Reprod., 5, 252-257.

Miller R. C., Fechiermer N. S., JaAp R. G., 1976. Distribution of karyotype abnormalities in chick embryo sibships. Biology of Reproduction, 14, 549-560.

Nalbandov A. V., Card L. E., I943. Effect of stale sperm on fertility and hatchability of chicken egigs. Poult. Sci., 22, $218-226$.

Parker J. E., 1950. The effect of restricted mating in flocks of new hampshire chickens on fertility and hatchability of eggs. Poult. Sci., 29, 268-270. 